\title{
Bullous Pemphigoid Masquerading as a Prosthesis Allergy
}

\author{
Darren J. Guffey, MD; Barrett J. Zlotoff, MD
}

\section{PRACTICE POINTS}

- Bullous pemphigoid frequently presents with urticarial plaques without classic tense blisters in the early phase of disease.

- The phenomenon of locus minoris resistentiae can lead to the presentation of bullous pemphigoid in locations traumatized by surgery.

- Bullous pemphigoid can present as urticarial plaques at surgery sites mimicking allergic contact dermatitis or reaction to surgical sutures or hardware.

To the Editor:

Bullous pemphigoid (BP) is an autoimmune bullous dermatosis characterized by tense subepidermal blisters. It primarily affects older individuals who typically report pruritus in the affected area. Subepidermal blisters are caused by a humoral and cellular autoimmune attack directed against 2 BP antigens-BP180 and BP230which are 2 critical components of the hemidesmosome whose primary function is to anchor the epidermis to the underlying dermis. Although tense bullae typically prompt immediate consideration of BP in the differential diagnosis, early disease often is characterized by urticarial plaques that require a high degree of suspicion to make the appropriate diagnosis. Locus minoris resistentiae is a term used to describe the phenomenon of skin disease occurring at the point of least resistance. ${ }^{1}$

A 79-year-old woman with type 2 diabetes mellitus, peptic ulcer disease, and hypertension was referred to the dermatology clinic due to concern for allergic contact dermatitis limited to the area of and adjacent to a wellhealed surgical wound. History and examination revealed that the patient had sustained a left femoral neck fracture 10 months prior to presentation that required closed reduction and surgical pinning. The surgical site healed well postoperatively; however, 7 months after surgery, she began to develop edema and erythema within and immediately adjacent to the surgical scar. She subsequently developed areas of superficial erosion within the erythema and was evaluated by her surgeon who was concerned for suture granuloma. Superficial wound debridement of the area was performed without improvement. Approximately 9 months after surgery, the patient developed bullae along the old surgical site, which raised concern for an allergic reaction to the implanted screws. Orthopedics elected to remove the hardware but also sent intraoperative tissue for pathologic examination, which revealed subepidermal bullae containing eosinophils and neutrophils, most consistent with a bullous drug eruption. During the ensuing weeks after hardware removal, the plaque spread along the old surgical wound, and several bullous lesions began to appear. The patient's primary care physician became concerned for allergic contact dermatitis, possibly to the surgical scrub employed during hardware removal. He prescribed triamcinolone ointment $0.1 \%$ and referred the patient to dermatology.

Upon presentation to dermatology, the patient noted stinging pain and intense pruritus of the affected area. Examination revealed a pink edematous plaque distributed along a well-healed surgical wound (Figure). Numerous fluid-filled tense bullae were superimposed on this plaque as well as areas of superficial erosion with serum crust. An expanded examination revealed similar smaller lesions on the upper arms, inner thighs, and lateral breasts. A 4-mm punch biopsy of lesional and perilesional skin was sent for hematoxylin and eosin staining

From the Department of Dermatology, University of Virginia, Charlottesville.

The authors report no conflict of interest.

Correspondence: Darren J. Guffey, MD, University of Virginia, Department of Dermatology, 1215 Lee St, PO Box 800718 , Charlottesville, VA 22908 (darrenjguffey@virginia.edu).

doi:10.12788/cutis.0398 

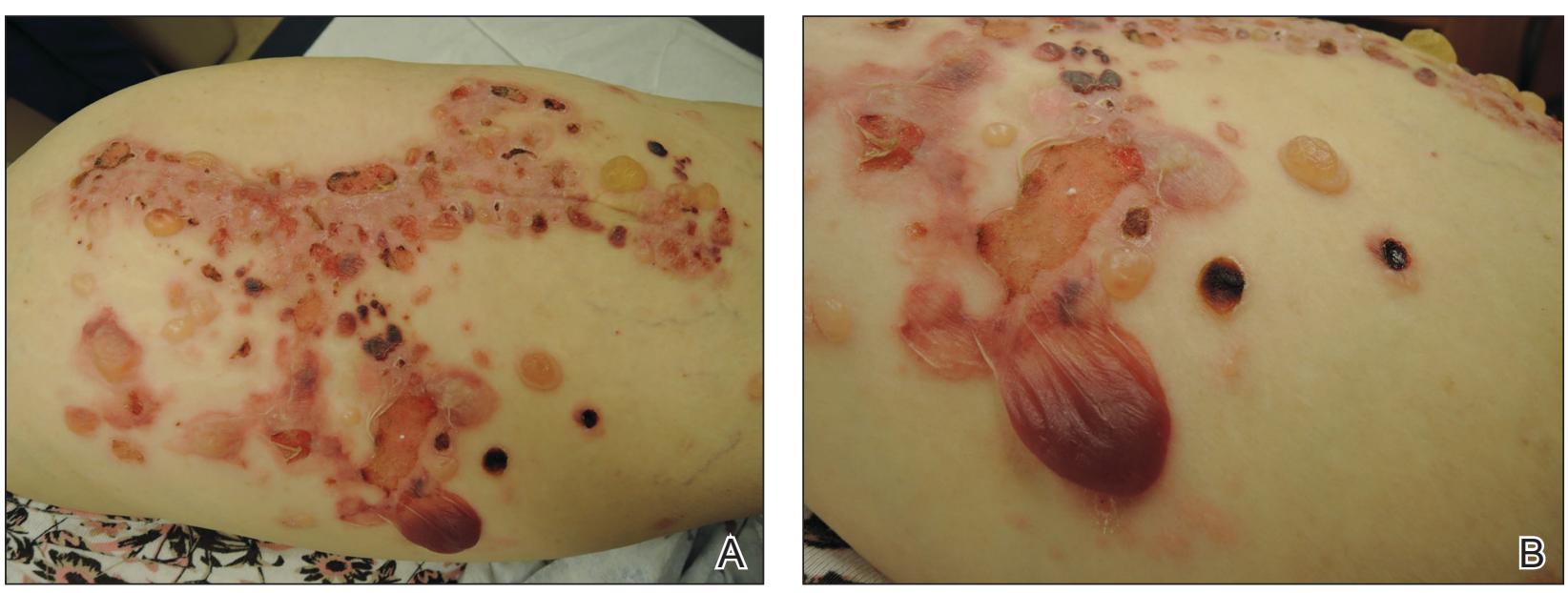

Bullous pemphigoid. A, Pink edematous plaque with superimposed tense bullae and erosions with serum crust on the left hip tracking along a well-healed surgical incision. B, Large tense bullae and erosion with serum crust arising within an edematous plaque.

and direct immunofluorescence, which demonstrated a subepidermal bullous dermatosis with a predominance of neutrophilic inflammation as well as a band of linear IgG deposition at the dermal-epidermal junction. The patient was diagnosed with $\mathrm{BP}$ exhibiting a locus minoris resistentiae phenomenon within the surgical site. She was started on prednisone $1 \mathrm{mg} / \mathrm{kg}$ daily and doxycycline $100 \mathrm{mg}$ twice daily and demonstrated rapid improvement.

Although the tense bullae seen in well-developed $\mathrm{BP}$ are fairly characteristic, the prodromal phase of this disease can present with urticarial plaques that are nonspecific. This progression is well described, but our case demonstrates the difficulty of considering BP when a patient presents with an urticarial plaque. As lesions progress to the bullous phase, they may be inappropriately diagnosed as allergic contact dermatitis, an error that may lead to unnecessary interventions (eg, removal of an implicated prosthesis). This case is a reminder that not all cutaneous eruptions in and around postsurgical scars are allergic in nature.

This case also depicts BP appearing in the locus minoris resistentiae, a well-healed surgical wound in our patient. Although many diseases have been shown to exhibit this type of isomorphic response, this phenomenon may pose diagnostic and management conundrums. Locus minoris resistentiae has been reported in many different diseases, both cutaneous and otherwise, but there likely are distinct disease- and case-specific mechanisms via which this occurs. Local phenomena reported to trigger BP include contact dermatitis, vaccination, radiation therapy, phototherapy, infection, and surgery. ${ }^{2}$ We suspect that the mechanism of locus minoris resistentiae in our patient was disruption of the architecture of the dermalepidermal basement membrane zone due to surgical trauma. Disruption of this architecture may have resulted in exposure of previously occult antigens, recognition by $\mathrm{T}$ cells, T-cell stimulation of autoantibody production by B cells, binding of autoantibodies to BP180, complement deposition, recruitment of inflammatory cells, release of proteinases, and degradation of BP180 and extracellular matrix proteins. ${ }^{2}$

\section{REFERENCES}

1. Lo Schiavo A, Ruocco E, Russo T, et al. Locus minoris resistentiae: an old but still valid way of thinking in medicine. Clin Dermatol. 2014;32:553-556.

2. Lo Schiavo A, Ruocco E, Brancaccio G, et al. Bullous pemphigoid: etiology, pathogenesis, and inducing factors: facts and controversies. Clin Dermatol. 2013;31:391-399. 